\title{
DUST IN PLANETARY NEBULAE
}

\author{
Patrick F. Roche, \\ Royal Observatory, \\ Blackford Hill, \\ Edinburgh EH9 3HJ. Scotland.
}

The presence of dust in planetary nebulae can be deduced in several ways - from the observed depletions of condensable elements, internal extinction and, most directly, through the detection of infrared emission from the dust grains. We know that there is a substantial amount of dust in planetary nebulae, and that a significant fraction of the total luminosity emerges in the infrared through thermal emission in most objects. However, a number of questions still largely remain unsolved, and perhaps the most pressing of these are that we do not yet have a satisfactory understanding of the ultraviolet, optical or infrared properties of the dust grains and we also do not yet know exactly where the emitting grains are located within the nebulae; for example, are they mixed with the ionized gas, or in neutral inclusions or perhaps in a disk around the central star?

In this report, the starting point will, appropriately enough, be the review by Barlow (1983) in the last IAU symposium devoted to planetary nebulae. Since then, a number of important developments that bear on the nature, effects and location of dust in planetaries have occurred, but the most dramatic has been the successful IRAS mission which has measured the infrared fluxes of hundreds of planetaries; this is discussed in detail by PreiteMartinez in this volume.

\section{Evidence of dust at short wavelengths}

The effects of dust on the visible and ultraviolet emission from planetary nebulae can be seen through absorption and scattering of light. However, in almost all objects studied, these effects are small and it is difficult to measure them with sufficiently accuracy to put useful limits on the nature of the grains responsible.

Firstly, the interstellar extinction must be properly accounted for, and in many objects this can be substantial so that the shape of the adopted extinction curve is crucial; any deviations caused by internal extinction will be difficult to establish. Attempts have been made to find differences in the intensities of emission lines arising in the approaching and receding edges of pn which could be attributed to internal extinction, using high resolution spectroscopy. In this case, possible asymmetries in the nebular shells render the results uncertain, but an approach using emission lines spanning the wavelength range $3100-6600$ A has been used by Doughty \& Kaler (1982) to minimise this problem. Ultraviolet resonance lines have very high scattering optical depths. with a correspondingly high probability of 
being absorbed by dust. The ratio of CIV $\lambda 1549$ /CIII $\lambda 2297$ can be used as a diagnostic of the dust absorption optical depth (see Seaton 1983) for objects in which a large fraction of carbon is in the form of $C^{3+}$.

Light scattered off dust grains may be visible in some objects. NGC 7027 has been known to have a faint reflection nebula extending over a diameter of 50 arcsec for some years (Atherton et al 1979), probably produced by dust scatttering in a massive neutral shell around the ionized zone. An extended optical halo with a diameter of up to 2 arcminutes has been detected around IC 418 by Monk, Barlow and Clegg (this meeting). The fact that the $[\mathrm{OII}] / \mathrm{H} \gamma$ line intensity ratio is the same in the halo as in the ionized nebula suggests that the halo results from dust grains reflecting the nebular emission lines. The picture for other objects is not as clear, for example conflicting reports on the extent of optical emission from Vy 2-2 were presented at this meeting by Clegg, Hoare and Walsh and by Falomo and Sabbadin. Scattering by dust grains within the ionized nebula can lead to measurable amounts of polarization. A recent study by Leroy et al (1986) has detected polarization of up to $1 \%$ in the [OIII] lines in $5 \mathrm{pn}$, which they attribute to scattering by dust particles.

Ionization structure models have now been developed that include dust grains heated by both trapped UV lines and direct heating by the central star and are able to account for the observed infrared emission (e.g. for NGC 7662 and NGC 3918, Harrington et al 1982, 1987). Further developments such as including the contributions from dust in neutral regions are underway (Hoare, this meeting), and synthesis of the nebular spectrum, taking into account absorption by dust grains, together with the infrared emission promises to give a clearer understanding of the dust in pn. Overall however, it appears that dust has a relatively small effect on the ionisation structure of the nebulae, although in order to account for the observations of UV resonance lines, there must be some dust present inside the ionized regions.

\section{The nature of dust in planetaries}

\subsection{Precursors}

We believe that the precursors of planetary nebulae are the asymptotic giant branch stars. These objects are typically cool stars immersed in thick circumstellar envelopes produced by heavy mass loss. From condensation theory (see e.g. Larimer 1979 and references therein), we expect these stars to condense copious amounts of dust in their circumstellar shells and indeed, this is what is observed. Different species of grain are produced according to the details of the stellar outflow, and in particular depending upon the chemical abundances of the circumstellar material. We therefore expect the grains in planetary nebulae to reflect the conditions present in the precursor wind, though the ionizing flux from the planetary nebula will affect the dust particles, which may in turn influence the development of the ionized nebula.

\subsection{Depletions}

It has been shown that elements, such as $\mathrm{Fe}, \mathrm{Mg}, \mathrm{Si}$, are underabundant in the gas phase compared to the expected values (Shields 1983); it is just these elements that would be 
expected to condense out as refractory grain species in planetary nebulae. However, the depletions of other species, notably carbon and oxygen, whose total abundances in the nebulae may be altered by nuclear processing, are much less certain. It is clear that carbon is not depleted by the factors of $10-20$ found for iron, but some depletion probably occurs, and in carbon-rich objects, some form of carbon may well account for most of the dust.

\subsection{Infrared observations of dust}

Twenty years ago, Gillett, Low \& Stein (1967) detected strong emission at $10 \mu \mathrm{m}$ from NGC 7027 and interpreted this as emission from warm dust. This has of course been confirmed, and the photometric surveys of Cohen \& Barlow $(1974,1980)$ showed that many pn display strong infrared emission from dust. Recently, near-infrared (1-4 $\mu \mathrm{m})$ photometry has been obtained by several groups (Whitelock 1985, Persi et al (1987) and Pena \& Torres-Peimbert at this meeting) pinpointing those nebulae that contain hot dust with colour temperatures up to $1000 \mathrm{~K}$. In addition to the hundreds of planetaries that have been measured photometrically by IRAS, mid-infrared spectra of about 70 objects have been collected from either ground-based observations (Aitken \& Roche 1982, Roche \& Aitken 1986, and references therein) or the low resolution spectrometer on IRAS (Pottasch et al 1986). As well as several bright ionic fine-structure lines, these observations show a number of resolved features attributed to emission from different dust species. A few objects have been measured spectroscopically near $30 \mu \mathrm{m}$ using the Kuiper airborne observatory (Forrest, Houck \& McCarthy 1981) and here, too, there is evidence of spectral structure due to solid state features in grains in some of the objects. At shorter wavelengths spectra of a few nebulae near $3 \mu \mathrm{m}$ have been published by Allen et al (1982) and Martin (1987), while Cohen et al (1986) and Wooden et al (1987) have presented spectra of 8 objects between 4-8 $\mu \mathrm{m}$.

Measurement of spectral features due to dust grains can, in principle, lead to the identification of the species producing the emission features and permit investigation of the chemistry of the nebular ejecta.

Principally through spectroscopy near $10 \mu \mathrm{m}$, it has been established that the infrared spectral signatures and therefore the composition of the dust grains vary from nebula to nebula. Broad features peaking at $9.7 \mu \mathrm{m}$ and $11.2 \mu \mathrm{m}$, were first seen in spectra of circumstellar dust shells around late type oxygen-rich and carbon-rich stars and were identified as emission from silicate and silicon carbide grains respectively on the basis of wavelength coincidence and the expected condensates in the different environments (e.g. Treffers \& Cohen 1974). It was therefore likely that the dust seen in planetary nebulae could also be designated as having formed from $\mathrm{O}$-rich or $\mathrm{C}$-rich environments where the silicate or $\mathrm{SiC}$ signatures were detected; this has been verified by abundance determinations made by careful modelling of optical and ultraviolet emission lines. All the objects that show strong silicate emission bands that have been modelled have been found to have $C / O<1$; conversely those planetaries with $\mathrm{SiC}$ emission have proved to have $C / O>1$ (see Seaton 1983). 


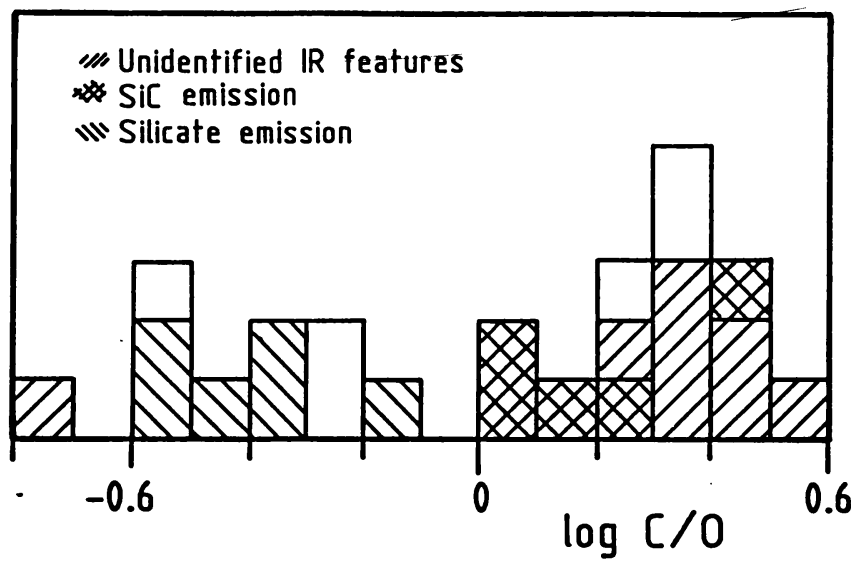

Fig 1. A histogram of those pn in the sample of Zuckerman \& Aller (1986) that have been observed at 8-13 $\mu \mathrm{m}$. Objects whose spectra are dominated by the different grain species are indicated by shading; the blank areas correspond to pn with weak continua at $10 \mu \mathrm{m}$.

However, of the objects for which good quality spectra near $10 \mu \mathrm{m}$ are available only about $40 \%$ show unambiguous evidence of either silicate or $\mathrm{SiC}$ emission. About half of the remainder show clear emission in the family of emission bands at $1^{1} 3,8.7$ and $7.7 \mu \mathrm{m}$ (and presumably also those at 6.2 and $3.3 \mu \mathrm{m}$ ) which were first detected in NGC 7027 (Gillett, Forrest \& Merrill 1973) whilst the rest have very weak continuum emission near $10 \mu \mathrm{m}$ and we are unable to identify clear spectral structure that could be due to dust. The family of emission bands between 3 and $13 \mu \mathrm{m}$ has come under intensive scrutiny in the last couple of years (see Leger, d'Hendecourt \& Boccara 1987 for comprehensive discussions) and it appears likely that they are produced by excitation of small $(.001 \mu \mathrm{m})$ carbon-rich grains, possibly the polycyclic aromatic hydrocarbons proposed by Duley \& Williams (1981) and Leger \& Puget (1984). Cohen et al (1986) found a good correlation between the strength of the $7.7 \mu \mathrm{m}$ band, the strongest of the family, and the $\mathrm{C} / \mathrm{O}$ ratio for a small sample of planetaries, such that the emission bands are more prominent where $\mathrm{C} / \mathrm{O}$ is higher. A similar conclusion has been reported by Martin (1987) from observations of the feature at $3.3 \mu \mathrm{m}$. This suggests that the grains giving rise to the family of emission bands are indeed carbon rich and would vindicate the suggestion of Barlow (1983) that those planetaries that are dominated by these features are very carbon rich with $\mathrm{C} / \mathrm{O}>2$.

It appears that, through the identification of spectral features and correlations with other observable quantities, we can classify the dust from planetaries as O-rich, C-rich or very C-rich. To illustrate this, figure 1 shows a histogram of those pn in the sample of Zuckerman \& Aller (1986) whose spectra have been measured at $10 \mu \mathrm{m}$. The different dust signatures seen in the infrared are indicated and it is clear that the qualitative estimates of $\mathrm{C} / \mathrm{O}$ obtained from the dust emission features are confirmed by the detailed gas-phase abundance determinations from the sources quoted by Zuckerman \& Aller. The only discrepant object is NGC 6302, a peculiar object with a very hot central star which shows 
the unidentified infrared bands, but also has a weak $\mathrm{OH}$ maser (Payne, Phillips \& Terzian in these proceedings) and has been reported to be oxygen-rich. The pn which have 10 $\mu \mathrm{m}$ continua that are too weak to allow classification are scattered across the histogram, implying that they include both carbon- and oxygen- rich objects.

From the excellent agreement between the gas-phase $\mathrm{C} / \mathrm{O}$ ratios and those predicted from the $10 \mu \mathrm{m}$ spectra, it is clear that our basic view of dust species in pn is correct. Furthermore, there are some planetaries which suffer so much interstellar extinction that accurate values of the $\mathrm{C} / \mathrm{O}$ ratio cannot be derived from $\mathrm{UV}$ and optical data. In these cases, the spectral features seen at 8-13 $\mu \mathrm{m}$ can be used to give rather more qualitative results; initial results of such a study of pn near the Galactic centre have been reported by Roche (1987).

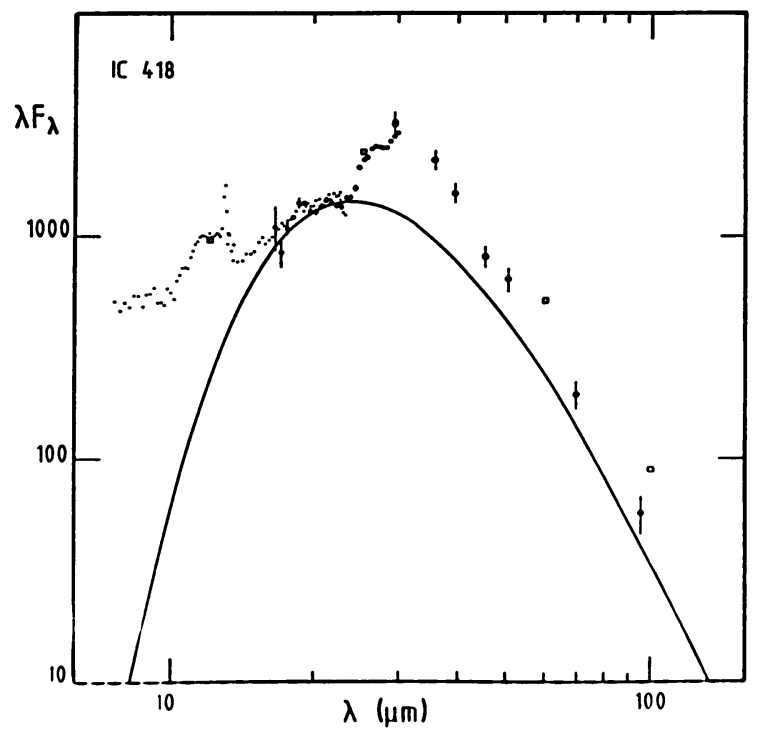

Fig 2. The infrared energy output from IC 418. The small dots shortwards of $22 \mu \mathrm{m}$ are from the IRAS LRS, the data between 17 and $30 \mu \mathrm{m}$ are from Forrest et al (1981), the data between 30 and $100 \mu \mathrm{m}$ are from Moseley $\&$ Silverberg (1986) and the open squares are from the IRAS survey. The solid line represents isothermal emission from grains with $Q=1 / \lambda^{2}$ at $\mathrm{T}=100 \mathrm{~K}$.

Although the $10 \mu \mathrm{m}$ spectral observations yield much valuable information about the warm $(300 \mathrm{~K})$ dust in pn, most of the dust is at considerably lower temperatures, and has not been studied in great detail. Spectra beyond $20 \mu \mathrm{m}$ are available for only four pn, NGC 7027, BD +30 3639, NGC 6572 and IC 418 (Forrest, Houck \& McCarthy 1981, Moseley \& Silverberg 1986), but the implications of the data are far reaching. In the first two objects, no clear spectral structure is apparent, but in IC 418 (figure 2) and NGC 6572, a strong broad emission feature, peaking near $30 \mu \mathrm{m}$ is present. This feature has also been measured in several carbon stars (Forrest et al 1981) and has been attributed to MgS (Goebel \& Moseley, 1985) possibly as a mantle on grains of some other composition. Moseley and 
Silverberg estimate that almost $25 \%$ of the total far-infrared flux is emitted in the "MgS" feature in IC 418 , which is a very large fraction for what is possibly a relatively minor dust component. Very little information is available on the spectral structure at wavelengths beyond $20 \mu \mathrm{m}$, and it is quite conceivable that other emission features could lie in the farinfrared region, so that estimates of quantities such as dust mass and temperatures derived from broad band photometry alone could be subject to considerable uncertainties.

\section{Location of dust in planetary nebulae.}

The precise location of dust grains within pn is still uncertain. It is important to determine where the dust is located as this gives information on questions such as whether it plays an important role in softening the radiation field in the ionized region by selective absorption of high energy photons, whether emission from dust grains is important as a coolant of the ionized gas and whether the mass of dust calculated from the IR emission can be compared with the mass of ionized gas in order to derive the dust to gas ratio. From the absorption of trapped UV resonance lines, we know that at least some dust is mixed with the ionized gas but it is possible that these are the warm grains emitting at wavelengths below $20 \mu \mathrm{m}$ rather than the bulk of the dust emitting in the far-infrared. A limited amount of spatial work has been carried out using both IRAS data and ground-based observations and these data generally show that the extent of the dust emitting region is similar, but not necessarily identical, to that of the ionized region.

Although the spatial resolution of IRAS was rather low for looking at compact objects, "super-resolution" deconvolution techniques can give information down to scales of the order of $15 \operatorname{arcsec}$ at $25 \mu \mathrm{m}$ and 30 arcsec at $60 \mu \mathrm{m}$ and results for about 20 pn have been presented by Hawkins and Zuckerman at this meeting. Lester, Harvey \& Joy (1986) have used maximum entropy deconvolution techniques on data from the Kuiper Airborne Observatory to look at the spatial structure of NGC 7027 in the far-infrared and conclude that most of the 50 and $100 \mu \mathrm{m}$ radiation comes from a region of similar size to the ionized zone and at most $50 \%$ larger. Unfortunately, the resolution attained is insufficient to clearly show whether the emitting dust lies inside, or just outside the ionized region.

Spatial distributions at 10 and $20 \mu \mathrm{m}$ can be measured with ground-based telescopes where 1-arcsec resolution is reachable. NGC 7027 was mapped by Becklin et al (1973) who found that the distribution of $10 \mu \mathrm{m}$ emission was very similar to that seen in high resolution radio maps demonstrating that the warm dust is well mixed with the gas in that object. A more complex picture emerged when Aitken \& Roche (1983) scanned across the minor axis of NGC 7027 with a grating spectrometer, simultaneously measuring the spatial structure in the $10.5 \mu \mathrm{m}$ [SIV] emission line, the $11.3 \mu \mathrm{m}$ narrow emission feature and the continuum emission at 10,11 12 and $13 \mu \mathrm{m}$. They showed that all the continua shared the same profile, ruling out a steep temperature gradient in the emitting dust, whilst the [SIV] profile was slightly narrower. In contrast, the scans in the $11.3 \mu \mathrm{m}$ emission feature had a profile whose FWHM is greater than that of the continuum by 1.5 arcsec, clearly showing that the grains emitting the $11.3 \mu \mathrm{m}$ feature lie outside the ionized zone and are probably contained in a neutral shell. The most natural explanation for these observations is that the grains that produce the narrow emission bands are destroyed in the ionized zone of NGC 7027; a similar conclusion has been reached in studies of the Orion ionization front 
where again, the dust emission features peak just outside the ionized region (Aitken et al 1979 , Sellgren 1981). A consequence of this is that the dust in a planetary nebula is likely to vary in both the composition of the species present and in the mean grain size between the ionized and neutral regions.

A different spatial distribution has been found in Abell 30 where Cohen \& Barlow (1974) found the $10 \mu \mathrm{m}$ emission to be centrally condensed. Observations by Dinerstein \& Lester (1984) revealed that the infrared emission from warm dust has a disk-like morphology, extended over about 20 arcsec, much less than the optical diameter. Lester \& Dinerstein (1984) also found an infrared disk at the centre of NGC 6302, coincident with a dark lane that crosses the nebula. The disk lies between the optical lobes with its long axis perpendicular to the long axis of the bipolar structure and probably plays some role in shaping the outflow. The compact nebula BD +303639 has been observed at 10 and 20 $\mu \mathrm{m}$ by Bentley et al (1984) who conclude that the dust emission is coextensive with emission from ionized hydrogen; higher spectral resolution scans across BD +30 3639 at $10 \mu \mathrm{m}$ were presented at this meeting by Smith et al who also conclude that the dust and gas emission come from the same volume, although the distributions of the two components may be different in detail.

The spatial distribution of near-infrared emission has been mapped out for several nebulae by scanning single detector photometers across the source. In most objects, the flux at wavelengths below $2 \mu \mathrm{m}$ is due to free-free emission and nebular emission lines, so that measurements at $\mathrm{J}$ and $\mathrm{H}$ trace out the distribution of ionized gas, although the central star may also contribute a significant signal in some sources. At longer wavelengths, emission from dust may become more prominent and observations near $4 \mu \mathrm{m}$ can delineate the emission from hot dust, while measurements through narrow-band filters at $3.3 \mu \mathrm{m}$ can follow the distribution of emission in the $3.3 \mu \mathrm{m}$ emission feature. Phillips et al (1984) found that in many cases, the near-infrared emission follows the ionized gas distribution, but in NGC 7027, after allowing for the the contribution from free-free emission, the $\mathrm{L}$ flux is more extended than the $\mathrm{J}, \mathrm{H}, \mathrm{K}$ and $\mathrm{M}$ scans. The interpretation is that the grains giving rise to the $3.3 \mu \mathrm{m}$ emission feature lie outside the ionized region, reflecting the results cited above at $11.3 \mu \mathrm{m}$. In the case of IC 418, Phillips et al (1986) found that a substantial fraction of the $2-4 \mu \mathrm{m}$ flux arises from a region considerably larger than the ionized nebula. In order to explain the high colour temperature of emission from dust in the neutral zone, they invoked a population of very small grains for which heating by absorption of single UV photons can give rise to high temperatures. It is just this mechanism that was proposed by Sellgren (1984) to account for the near-infrared properties of reflection nebulae, and extended by Leger and Puget (1984) to include the narrow emission features, and it is probable that the $3.3 \mu \mathrm{m}$ emission band in IC 418 measured by Russell, Soifer \& Merrill (1976) is produced in this way.

It is clear that there is no single answer to the question of where dust in planetary is located. In some objects, disk-like structures are evident where there is a substantial amount of dust girdling the central star. In others, the dust appears to be well mixed with the ionized gas, but it seems certain that the emission from some grain species is seen only, or at the least predominately, from neutral shells. 


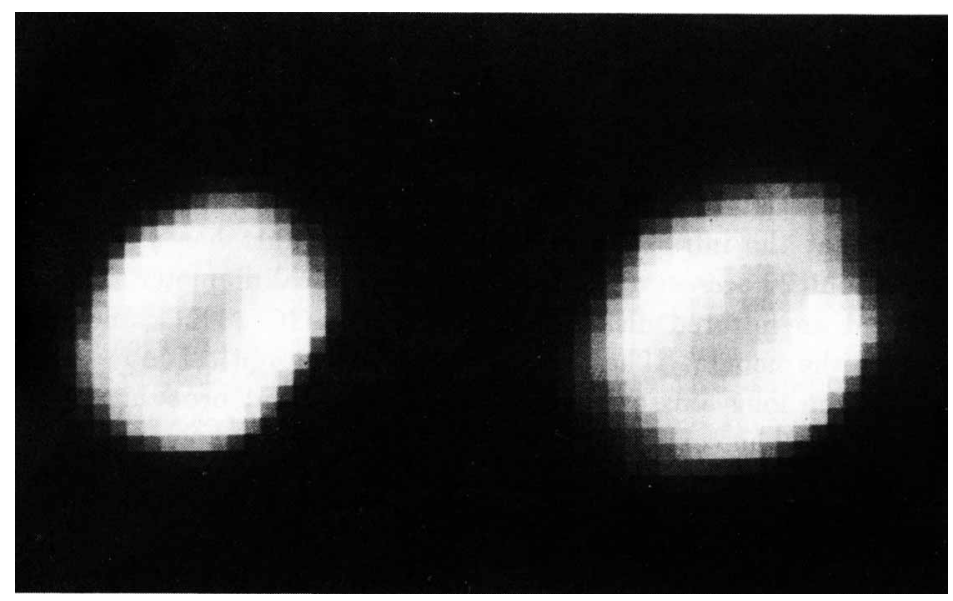

Fig 3. Images of NGC 7027 taken through filters at 2.17 (left) and $3.3 \mu \mathrm{m}$ (right) with the infrared camera at UKIRT. The pixel size is $0.6 \times 0.6$ "

\section{Infrared Imaging.}

With the release of sensitive infrared arrays, true imaging instruments are now available in the 1-5 $\mu \mathrm{m}$ spectral region. The first results on the spatial distribution of the $3.3 \mu \mathrm{m}$ dust emission and HII in NGC 7027 made with array detectors have appeared (Woodward et al 1987). Figure 3 shows grey scale representations of the emission from NGC 7027 taken with the UKIRT common-user infrared camera (IRCAM) at $2.17 \mu \mathrm{m}$ (Brackett $\gamma$ ) and $3.3 \mu \mathrm{m}$. It is immediately apparent that the $3.3 \mu \mathrm{m}$ image (where most of the flux is from the 3.3 $\mu \mathrm{m}$ dust feature with small contributions from Pfund $\delta$, free-free emission and continuum dust emission) is much more extended than the ionized gas traced by the $2.17 \mu \mathrm{m}$ image, consistent with the data of Woodward et al, and confirming the earlier work of Aitken \& Roche (1983) and Phillips (1984).

In $\mathrm{BD}+303639$, shown in fig 4 as a contour map, the IRCAM images clearly separate the central star from the ionized gas and show a somewhat different result. It appears that the $\mathrm{Br} \gamma$ and $3.3 \mu \mathrm{m}$ emission come from a region of similar size, but that there are differences in the detailed distribution. In particular, the $3.3 \mu \mathrm{m}$ emission is strongest in the north-eastern edge, and shows local minima at the positions of the most intense $\mathrm{Br} \gamma$ emission. It therefore appears that in this object, which has a relatively cool central star and low flux of hard photons, the grains that emit the narrow features are located within the same volume as the ionized gas, but are located in regions away from the maximum photon flux. Clearly the spatial distribution of the grains emitting at $3.3 \mu \mathrm{m}$ is not symmetric and it would be of interest to look at the nebular conditions in detail and at high spatial resolution to determine the conditions in the region where the $3.3 \mu \mathrm{m}$ emission peaks. 


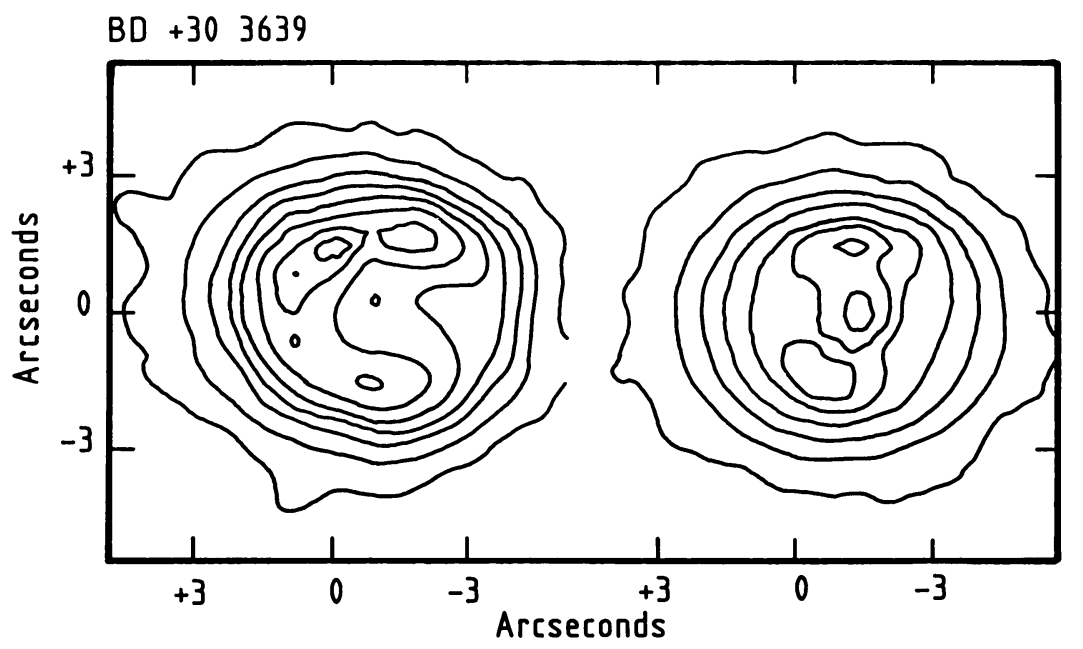

Fig 4. Contour maps of images of $B D+303639$ taken through filters at 3.28 (left) and $2.17 \mu m$ (right).

\section{Cold dust}

Dust which is warmer than about $30 \mathrm{~K}$ will have been seen by IRAS, but it is possible that there could be a substantial amount of dust outside the ionized regions which may be cooler than this; dust at $10 \mathrm{~K}$ emits very weakly in the far-infrared, but strongly in the submillimetre spectral region. In the last few months, two sub-mm dishes, the $15-\mathrm{m}$ JCMT and the 10-m Caltech Dish, have been opened on Mauna Kea in Hawaii allowing sensitive observations in the $300 \mu \mathrm{m}-2 \mathrm{~mm}$ region. Once these dishes are working to their design specifications, many planetary nebulae will come within their sensitivity limits for continuum observations, but the first detections of pn at 800 and $1100 \mu \mathrm{m}$ have already been made with the JCMT (Clegg et al private communication). Using UKIRT, Gee et al (1984) measured NGC 7027 at 350, 800 and $1100 \mu \mathrm{m}$ and found that the sub-mm flux was considerably higher than a simple extrapolation of the far-infrared data would predict. They interpreted this result as evidence of emission from cold dust in the massive neutral halo surrounding the ionized nebula. Observations of a number of $\mathrm{pn}$ at sub-mm wavelengths are under way (by Clegg et al and Kwok) and promise to pinpoint those objects surrounded by cold dust shells, and allow determinations of the mass of cold dust. A particular advantage of observations in the sub-mm where the grains are emitting in the Rayleigh-Jeans limit, is that the determination of the mass of dust depends linearly upon the black body function at the adopted temperature and the emissivity of the grains (e.g. Hildebrand 1983).

\section{Summary}

An understanding of the composition and spatial distribution of dust is vital in order for us to be able to interpret the infrared emission from pn. In particular, care is required 
when looking at large samples of nebulae as estimates of quantities such as absorption cross section, emissivity and grain size and hence the mass of dust, depend upon the adopted grain species whilst the derived gas to dust ratio requires knowledge of whether or not the bulk of the dust lies within the ionized region. Whilst our present understanding of these questions is inadequate, much progress can be made in answering these questions using the new infrared and sub-mm instrumentation currently under development at various observatories, although we will probably have to wait until the launch of ISO to investigate the far-infrared spectral properties of dust grains in detail. Within the next few years, we may expect sensitive infrared cameras operating in the 10 and $20 \mu \mathrm{m}$ atmospheric windows to become available, and we will then be in a position to trace the spatial distribution of dust at temperatures down to $100 \mathrm{~K}$, which is approaching the temperature of the bulk of the dust in many pn. Finally, there is no doubt that planetary nebulae offer some of the best opportunities for unravelling the properties of cosmic dust as many pn are bright objects whose history and composition are relatively well understood.

\section{References :}

Aitken, D.K. \&oche, P.F., 1982. MNRAS., 200, 217.

Aitken, D.K. \& Roche, P.F., 1983. MNRAS., 202, 1233.

Aitken, D.K., Roche, P.F., Spenser, P.M. \& Jones, B, 1979. Astr. Ap., 76, 60.

Allen, D.A., Baines, D.W.T., Blades, J.C. \& Whittet, D.C.B., 1982. MNRAS, $199,1017$.

Atherton, P.D., Hicks, T.R., Reay, N.K., Robinson, G.J., Worswick, S.P. \& Phillips, J.P., 1979. Ap.J., 232, 786.

Barlow, M.J., 1983. IAU Symp 103, "Planetary Nebulae", ed D.R. Flower, p105.

Becklin, E.E., Neugebauer, G. \& Wynn-Williams, C.G., 1973. Ap.Lett., 15, 87.

Bentley, A.F., Hackwell, J.A., Grasdalen, G.L. \& Gehrz, R.D., 1984. Ap.J., 278,665 .

Cohen, M., Allamandola, L., Tielens, A.G.G.M., Bregman, J., Simpson, J.P., Witterborn, F.C., Wooden, D. \& Rank, D., 1986. Ap.J., 302, 737.

Cohen, M. \& Barlow, M.J., 1974. Ap.J., 193, 401.

Cohen, M. \& Barlow, M.J., 1980. Ap.J., 238, 585.

Dinerstein, H.L. \& Lester, D.F., 1984, Ap.J., $281,702$.

Doughty, J.R. \& Kaler, J.B., 1982. PASP, 94, 43.

Duley, W.W. \& Williams, D.A., 1981. MNRAS, 196, 269.

Gee, G., Emerson, J.P., Ade, P.A.R., Robson, E.I. \& Nolt, I.G., 1984. MNRAS, 208, 517 .

Forrest, W.J., Houck, J.R. \& McCarthy, J.F., 1981. Ap.J. 248, 195.

Gillett, F.C., Forrest, W.J. \& Merrill, K.M., 1973. Ap.J., 183, 87.

Gillett, F.C., Low, F.G. \& Stein, W.A., 1967. Ap.J., 149, L97.

Goebel, J.H. \& Moseley, H., 1985. Ap.J. 290, L35.

Harrington, J.P., Seaton, M.J., Adams, S., Lutz, J.H., 1982. MNRAS, 199, 517.

Harrington, J.P., Monk, D.J. \& Clegg, R.E.S., 1987. MNRAS in press.

Hildebrand, R.H., 1983. Q.Jl. R.A.S., 24, 267.

Larimer, G.W., 1979. Astr. Sp. Sci., 65, 351. 
Leger, A., d'Hendecourt, L. \& Boccara, N., 1987. "Polcyclic Aromatic Hydrocarbons and Astrophysics", Reidel, Dordrecht.

Leger, A. \& Puget, J.L., 1984. Astr. Ap., 137, L5.

Leroy, J.L., Le Borgne, J.F. \& Arnaud, J., 1986. Astr. Ap., 160, 171.

Lester, D.F., Harvey, P.M. \& Joy, M., 1986 Ap.J., 304, 623.

Lester, D.F. \& Dinerstein, H.L., 1984. Ap.J., 281, L67.

Martin, W., 1987. Astr. Ap., 182, 290.

Moseley, H. \&ilverberg, R.F., 1985. "Interrelationships among circumstellar, Interstellar and Interplanetary Dust." ed J.A. Nuth \& R.E. Stencel. p 233. NASA CP-2403.

Persi, P., Preite-Martinez,A., Ferrari-Toniolo, M., \& Spinoglio, L., 1987. "Planetary and protoplanetary nebulae:From IRAS to ISO." p 221.

Phillips, J.P., Mampaso, A., Vilchez, J.M. \& Gomez, P., 1986. Astr. Sp. Sci., 122, 81.

Phillips, J.P., Sanchez Magra, C. Martinez Roger, C., 1984. Astr. Ap., $133,395$.

Pottasch, S.R., Preite-Martinez, A., Olnon, F.M., Jing-Er, Mo \& Kingma, S., 1986, Astr. Ap., 161, 363.

Roche, P.F. 1987. Proc. Vulcano Workshop "Planetary and proto-planetary nebulae: From IRAS to ISO." p 45. ed A. Preite-Martinez. Reidel.

Roche, P.F. Aitken, D.K., 1986. MNRAS, 221, 63.

Russell, R.W., Soifer, B.T. \& Merrill, K.M., 1977. Ap.J., 213, 66.

Seaton, M.J., 1983. IAU Symp 103, "Planetary Nebulae", ed D.R. Flower, p129.

Sellgren, K., 1981. Ap.J., 245, 138.

Sellgren, K., 1984. Ap.J., 277, 623.

Shields, G.A., 1983. IAU Symp 103, "Planetary Nebulae", ed D.R. Flower, p259.

Treffers, R.R. Cohen, M., 1974. Ap.J., 188, 545.

Whitelock, P.A., 1985. MNRAS, 213, 59.

Wooden, D.H., Cohen, M., Bregman, J.D., Witterborn, F.C., Rank, D.M., Allamandola, L.J. \& Tielens, A.G.G.M., 1986. "Proc. Summer School on Inter-stellar Processes." p 59. ed. D.J. Hollenbach \& H.A. Thronson, NASA TM-88342.

Woodward, C.E., Pipher, J.L., Shure, M.A., Forrest, W.J., Sellgren, K. \&

Nagata, T., 1987. "Infrared Astronomy with Arrays", Proc. Hilo Detector

Workshop, p 299. ed C.G. Wynn-Williams \& E.E. Becklin. Univ. Hawaii.

Zuckerman, B. Aller, L.R., 1986. Ap.J., 301, 772. 


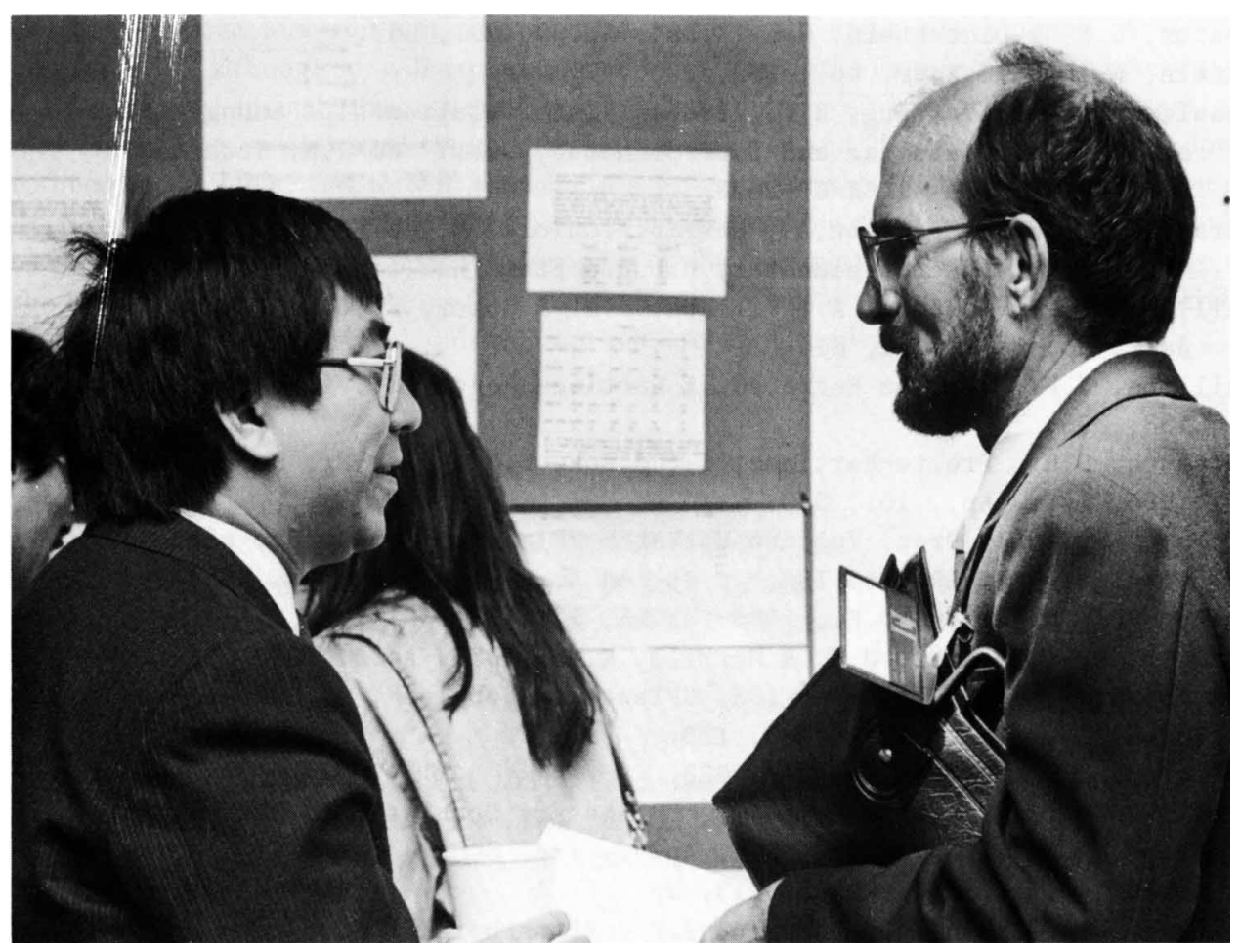

Sun Kwok and Bob O'Dell. 